Macedonian Pharmaceutical Bulletin, 66 (Suppl 1) 135 - 136 (2020)

Online ISSN 1857 - 8969

UDC: 615.453 .6 .015 .152

DOI: 10.33320/maced.pharm.bull.2020.66.03.067

Short communication

\title{
Effect of binder, lubricant and compression force levels on hardness and disintegration time of immediate release tablets with a high-dose API - a $3^{3}$ full-factorial design
}

\author{
Krume Toshev*, Natasha Anevska Stojanovska, Sonja Ugarkovic
}

Alkaloid AD, Aleksandar Makedonski 12, 1000 Skopje, RN Macedonia

\section{Introduction}

The skills of the formulation scientist are often put at test when a very high-dose API (>95\%) with unfavorable properties is to be incorporated in an immediate release tablet. As the room for excipients in tablets with $>95 \%$ API is so small, a very careful consideration should be made on the choice and concentration of each of the excipients and the parameters of the process in order to achieve desirable properties of the tablets (Lionberger, 2008).

The aim of this study is to evaluate the effect of the levels of binder, lubricant and compression force on the hardness and disintegration time of immediate release tablets with API of BCS class III present in the formulation at a very high-dose $(>95 \%)$.

\section{Materials and methods}

API of BCS class III is an oral hypoglycemic agent that has very poor flow and compression properties and ability to form hard lumps with storage. The other excipients in the formulation are binder - povidone K90 and lubricant - magnesium stearate. Due to the unfavorable properties of the API the chosen manufacturing process was wet granulation.
In this study a $3^{3}$ full-factorial response surface design with 30 experiments (including 3 central points) is used to evaluate the effects of levels of binder - povidone K90, lubricant - magnesium stearate and compression force on hardness and disintegration time of the immediate release tablets. (Armstrong 2006). The tested levels in the formulation for the binder povidone $\mathrm{K}-30$ are $3 \%$, $4 \%$ and $5 \%$, the tested levels for the lubricant are $1 \%, 1.5 \%$ and $2 \%$, and the tested main compression force levels are $10 \mathrm{kN}, 15 \mathrm{kN}$ and $20 \mathrm{kN}$.

For the purpose of the study, small laboratory trials of $0.5 \mathrm{~kg}$ were made. API was obtained from manufacturer USV. API was wet granulated with the binder povidone K90 on a fluid-bed granulator after which the lubricant is added extragranularly. Each of the trials was compressed at 3 different compression forces $10 \mathrm{kN}, 15 \mathrm{kN}$ and $20 \mathrm{kN}$ on a Korsch XL 100 Pro tablet press. The disintegration time was tested on apparatus for disintegration Erweka ZT322 and the hardness was tested on a hardness tester Erweka TBH 425TD.

Evaluation of raw data and model interpretation was performed using MODDE Go ${ }^{\circledR}$ statistical software. In order to predict the variability of the results for response variables with varying the values for independent variables, Multiple linear regression (MLR) was used.

\footnotetext{
*ktosev@alkaloid.com.mk
} 


\section{Results and discussion}

The chosen model (quadratic model) was shown to be significant and fitting for the data for all responses. The predictive ability of the model is moderate to high for all responses. The observed vs. predicted response values of the responses plot shows that most results fall on the 1:1 line which indicates good model.

From the effect plots the highest effects of each of the factors binder, lubricant and the compression force were observed when the other two factors are set at medium level. The highest effect on the response hardness is from the binder povidone K90 and the compression force. The highest effect on the response disintegration time is from the lubricant magnesium stearate. We can also observe that tablet hardness increases with increasing compression force from $10-20 \mathrm{kN}$ and with increasing \% of binder povidone $\mathrm{K} 90$ in formulation from $3-5 \%$.

Disintegration time prolongs with increasing magnesium stearate $(\%)$ from 1-2\%. Disintegration time is not affected by change in compression force or binder $(\%)$. From the response surface plots the interaction of two factors can be studied when the third factor is set at medium level and their effect on the disintegration and hardness of tablets. A sharp increase in hardness can be observed when $\%$ of binder povidone $\mathrm{K} 90$ in formulation increases from $3-5 \%$, the compression force is at its max $20 \mathrm{kP}$ and the $\%$ of magnesium stearate is at its median level $1.5 \%$. The same sharp increase is also observed when the compression force increases from $10-20$ $\mathrm{kN}$ with the $\%$ of binder povidone $\mathrm{K} 90$ in formulation set at the $\max 5 \%$ and the $\%$ of magnesium stearate is at its median level $1.5 \%$ which showcases the additive effect of the compression force and the $\%$ of binder on the hardness of tablets. The lubricant magnesium stearate, on the other hand, in levels from $1 \%$ to $1.5 \%$ has very small positive effect on the hardness which turns into a negative effect when the level is increased to $2 \%$. The disintegration time decreases sharply when the $\%$ of magnesium stearate decreases from $2 \%$ to $1 \%$, with a very little additive effect of the compression force. It is interesting to observe that the effect of the lubricant is more pronounced on the disintegration time of the tablets than the effect of the compression force. The formulation can be further optimized to have the fastest disintegration times with the highest hardness, and also a sweet spot can be made to cover the areas where the responses are within desired ranges. From the optimization of the formulation tool in the MODDE Go ${ }^{\circledR}$ statistical software, in order to achieve tablet with highest hardness and fastest disintegration time, the chosen formulation should contain $4.1 \%$ povidone K90, $1.2 \%$ magnesium stearate and should be compressed at $20 \mathrm{kN}$ main compression force.

\section{Conclusion}

In order to achieve desirable properties of the tablets especially in formulations where the room for excipients is very low it is of crucial value that the excipients and their quantities are chosen wisely and the parameters of the process are set properly. The $3^{3}$ full-factorial design adequately describers the correlation of the levels of binder, lubricant and compression force and their main and interaction effects on the hardness and disintegration time of immediate release tablets with API of BCS class III.

The highest positive effects on the response hardness can be observed from the binder povidone K90 and the compression force and the highest negative effect on the disintegration time can be observed from the lubricant magnesium stearate. Additive interaction effect can be observed of the compression force and the $\%$ of binder on the hardness of tablets. The disintegration time is mostly affected by the lubricant magnesium stearate with a negligible additive effect of the compression force.

The formulation can be further optimized to achieve the fastest disintegration time with the highest hardness.

\section{References}

Armstrong, N.A., James, K.C., 2006. Pharmaceutical experimental design and interpretation, $1^{\text {st }}$ ed., Taylor and Francis, London.

Lionberger, R.A., 2008, FDA critical path initiatives: opportunities for generic drug development. The AAPS Journal 10(1), 103-109.

Maced. Pharm. Bull. 66 (Suppl 1) 135 - 136 (2020) 